\title{
An Approach for Solving Discrete Game Problems with Total Constraints on Controls
}

\author{
Asqar Raxmonov' ${ }^{1}$ and Gafurjan I. Ibragimov ${ }^{2}$ \\ ${ }^{1}$ Department of Informatics, Tashkent University of Information Technologies (TUIT), Amir Temur Street 108, \\ 100202 Tashkent, Uzbekistan \\ ${ }^{2}$ Institute for Mathematical Research and Department of Mathematics, Faculty of Science, Universiti Putra Malaysia, \\ 43400 Serdang, Selangor, Malaysia
}

Correspondence should be addressed to Gafurjan I. Ibragimov; ibragimov@upm.edu.my

Received 12 November 2013; Revised 5 March 2014; Accepted 11 March 2014; Published 17 April 2014

Academic Editor: Valery Y. Glizer

Copyright (C) 2014 A. Raxmonov and G. I. Ibragimov. This is an open access article distributed under the Creative Commons Attribution License, which permits unrestricted use, distribution, and reproduction in any medium, provided the original work is properly cited.

\begin{abstract}
We consider a linear pursuit game of one pursuer and one evader whose motions are described by different-type linear discrete systems. Controls of the players satisfy total constraints. Terminal set $M$ is a subset of $\mathbb{R}^{n}$ and it is assumed to have nonempty interior. Game is said to be completed if $y(k)-x(k) \in M$ at some step $k$. To construct the control of the pursuer, at each step $i$, we use positions of the players from step 1 to step $i$ and the value of the control parameter of the evader at the step $i$. We give sufficient conditions of completion of pursuit and construct the control for the pursuer in explicit form. This control forces the evader to expend some amount of his resources on a period consisting of finite steps. As a result, after several such periods the evader exhausted his energy and then pursuit will be completed.
\end{abstract}

\section{Introduction}

A large number of works are devoted to differential games where the position of the players changes continuously in time (see, e.g., [1-18]). Zero-sum differential games were first considered in the book of Isaacs [5] who derived the main equation of the theory of differential games. Though the Isaacs method is not complete (since the main equation may not have classical solutions or may have infinitely many generalized solutions [18]) he effectively applied it to solve many interesting game problems.

Krasovskiı and Subbotin [7] and Pontryagin [10] proposed two different formalizations for differential games. According to Pontryagin we have to identify ourselves with the pursuer in pursuit games and with the evader in evasion games. Many approaches have been proposed in literature to solve differential game problems under the integral constraints (see, e.g., $[1-4,6,8,9,11-15,17]$ ).

In the present paper, we study a linear pursuit game with total constraints on controls. Such constraints are discrete analogues of integral constraints for differential games.
There are a few papers that study discrete games under total constraints (see, e.g., [14-16, 19-22]).

In the linear discrete game studied by Satimov et al. [14] , the eigenvalues of the main matrix are assumed to be real. Some sufficient conditions of completion of pursuit were obtained in this paper. In the paper of Satimov et al. [15], motions of players are simple. It was a starting point for multiperson differential games with integral constraints. Some sufficient conditions were obtained for the game to be terminated.

In the paper of Ibragimov [20], a discrete game described by the equation

$$
x(k+1)=A x(k)-u(k)+v(k)
$$

is studied. Control of the evader satisfies total constraint. There are two game problems that are considered. In the first game, the control of the pursuer satisfies geometric constraint, and in the second it satisfies total constraint. Sufficient conditions of completion of game from any position were obtained. 
Azamov and Kuchkarov [19] studied relationship between 0 -controllability of linear discrete systems

$$
x(k+1)=A x(k)+B u(k)
$$

and completion of linear pursuit discrete game described by the equation

$$
z(k+1)=A z(k)+B u(k)+C v(k) .
$$

The control of the pursuer is subjected to geometric constraint and that of the evader is subjected to total constraint. Necessary and sufficient conditions were obtained under which solvability of 0 -controllability is equivalent to completion of pursuit.

Kuchkarov et al. [22] studied a discrete game whose position $z(k+1) \in \mathbb{R}^{n}$ is described by the above equation. Different from the above game, both controls of the players are subjected to total constraints. They proved that if eigenvalues of the matrix $A$ in absolute value are less than 1 and $\operatorname{dim} B S=n$, where $S$ is unit ball in $\mathbb{R}^{n}$ centered at the origin, then pursuit can be completed from any initial position. This result much more improves the result of the work by Ibragimov and Kuchkarov [21].

In the present paper, we study a linear pursuit discrete game of one pursuer and one evader. It is assumed that total constraints are imposed on controls of players. The main point in the pursuit method is that, in one step, the pursuer forces the evader to expend a certain amount of his resources to prevent the game from being completed. Therefore, after finite times of such steps the resources of the evader will be exhausted, and then pursuit will be completed. We obtain sufficient conditions of completion of pursuit.

\section{Statement of the Problem}

In the Euclidian space $\mathbb{R}^{n}$, we consider a discrete game described by the following equations:

$$
\begin{aligned}
& x(k)=A x(k-1)+u(k), \\
& y(k)=B y(k-1)+v(k),
\end{aligned}
$$

where $x, y, u, v \in \mathbb{R}^{n}, n \geq 1, A$ and $B$ are $n \times n$ constant matrices, and $u$ (resp., $v$ ) is control parameter of the pursuer (evader). The parameters $u$ and $v$ are chosen in the form of sequences as follows:

$$
\begin{gathered}
u=u(\cdot)=\{u(1), u(2), \ldots, u(k), \ldots\} \\
v=v(\cdot)=\{v(1), v(2), \ldots, v(k), \ldots\}
\end{gathered}
$$

and subjected to the following constraints:

$$
\begin{gathered}
\|u(\cdot)\|_{l_{2}} \leq \rho, \\
\|v(\cdot)\|_{l_{2}} \leq \sigma,
\end{gathered}
$$

where $\rho$ and $\sigma$ are positive numbers. The pursuer and evader move according to (4) and (5), respectively. In the space $\mathbb{R}^{n}$, a terminal set $M$, whose interior is not empty, is defined.
The condition int $M \neq \varnothing$ implies that there are a number $\ell>0$ and vector $m \in M$ to satisfy the inclusion $\ell S \subset-m+M$. The purpose of the pursuer is to realize the inclusion

$$
y(k)-x(k) \in M
$$

at some finite step $k$, and that of the evader is opposite.

Definition 1. A sequence $u=u(\cdot)$ (resp., $v=v(\cdot)$ ) subjected to the constraint (7) (resp., (8)) is called admissible control of the pursuer (evader).

Definition 2. If for any admissible control of the evader $v=$ $v(\cdot)=\{v(1), v(2), \ldots, v(k), \ldots\}$ one can construct an admissible control of the pursuer $u(\cdot)=\{u(1), u(2), \ldots, u(k), \ldots\}$ such that for the solutions

$$
\begin{aligned}
& x=x(\cdot)=\{x(1), x(2), \ldots, x(k)\}, \\
& y=y(\cdot)=\{y(1), y(2), \ldots, y(k)\}
\end{aligned}
$$

of (4) and (5) with the initial positions $z_{0}=\left(x_{0}, y_{0}\right)$, where $x(0)=x_{0}, y(0)=y_{0}, y_{0}-x_{0} \notin M$, and controls $u(\cdot), v(\cdot)$ the inclusion (9) is satisfied at some $k \leq N\left(z_{0}\right)$, then we say that pursuit starting from the position $z_{0}$ can be completed in the game (4), (5) for $N=N\left(z_{0}\right)$ steps. Here, we assume that the pursuer uses $v(k)$ to construct $u(k), k \geq 1$.

Problem. Find sufficient conditions, under which pursuit can be completed from any initial position $z_{0}=\left(x_{0}, y_{0}\right), x_{0}, y_{0} \in$ $\mathbb{R}^{n}, y_{0}-x_{0} \notin M$.

\section{Main Result}

In this section, we formulate and prove the main result of the paper. First of all, we state our basic assumption. Let the norm of the matrix $A$ be defined by $\|A\|=\max _{|x|=1}|A x|$.

Assumption 3. There exist numbers $\rho_{1}>0, \rho_{2}>0, d>0,0 \leq$ $\alpha_{1}<1,0 \leq \alpha_{2}<1 / 2$, a positive integer $m$, and vector $m_{1} \in M$ such that $\rho_{1}+\rho_{2}=\rho$ and

(1) for any $x, y \in \mathbb{R}^{n}, y-x \notin M$, there exists a step $k=$ $k(x, y)>0$ such that

$-m_{1}+B^{k} y-A^{k} x \in \frac{\rho_{1}}{\sqrt{m \cdot k}}\left(A^{k-1}+A^{k-2}+\cdots+E\right) S ;$

(2) there exist linear operators $F(k, i): R^{n} \rightarrow R^{n}, 1 \leq i \leq$ $k$, such that for any $k \geq 1$ the following inequalities hold true:

(a) $\sum_{i=1}^{k}\left\|B^{k-i}-A^{k-i} F(k, i)\right\|^{2} \leq d m^{\alpha_{1}}$,

(b) $\|F(k, i)\| \leq \rho_{2} /\left(\sigma \cdot m^{\alpha_{2}}\right), 1 \leq i \leq k$;

(3) $m \cdot \min \left\{\sigma^{2} / m^{1-2 \alpha_{2}}, \ell^{2} /\left(d \cdot m^{\alpha_{1}}\right)\right\}>\sigma^{2}$.

Under this assumption we prove the following statement.

Theorem 4. If Assumption 3 holds, then pursuit can be completed in the game (4), (5) from any initial position $z_{0}=$ $\left(x_{0}, y_{0}\right), x_{0}, y_{0} \in \mathbb{R}^{n}, y_{0}-x_{0} \notin M$, for a finite step. 
Proof. Let $y_{0}-x_{0} \notin M$. Then it follows from condition (1) of Assumption 3 that there exists $k_{1}=k_{1}\left(x_{0}, y_{0}\right)>0$ such that

$$
\begin{aligned}
-m_{1}+B^{k_{1}} y_{0}-A^{k_{1}} x_{0} \in & \frac{\rho_{1}}{\sqrt{m \cdot k_{1}}} \\
& \times\left(A^{k_{1}-1}+A^{k_{1}-2}+\cdots+A+E\right) S .
\end{aligned}
$$

Consider the equation

$$
\left(A^{k_{1}-1}+A^{k_{1}-2}+\cdots+A+E\right) w=-m_{1}+B^{k_{1}} y_{0}-A^{k_{1}} x_{0},
$$

with respect to the unknown vector $w \in\left(\rho_{1} / \sqrt{m k_{1}}\right) S$. According to (12), (13) has a solution. Let $w=\widehat{w}$ be the lexicographically minimum solution of (13).

We now construct a control for the pursuer. Let the evader use an arbitrary admissible control $v=v(\cdot)=\{v(1)$, $v(2), \ldots, v(k), \ldots\}$; that is, behavior of the evader is any.

By Definition 2, the pursuer may use $v(i)$ to construct $u(i)$ on each step $i$. Set

$$
u(i)=F\left(k_{1}, i\right) v(i)+\widehat{w}, \quad i=1,2, \ldots,
$$

while

$$
\sum_{i=1}^{k}|v(i)|^{2}<\frac{\sigma^{2}}{m^{1-2 \alpha_{2}}}
$$

where $k$ is an integer. Then it is natural to consider the following three cases.

Case 1 . The inequality (15) holds for all $k \in\left\{1,2, \ldots k_{1}\right\}$.

Case 2. At some $k=n_{1}, n_{1}<k_{1}$, the inequality sign in (15) turns to equality.

Case 3. At some $k=n_{1}-1, n_{1}<k_{1}$, the inequality (15) holds, but at $k=n_{1}$ it fails to hold and opposite inequality holds:

$$
\sum_{i=1}^{n_{1}}|v(i)|^{2}>\frac{\sigma^{2}}{m^{1-2 \alpha_{2}}} .
$$

It follows from (4) and (5) that for the solutions $x(k)$ and $y(k)$ corresponding to the initial position $\left(x_{0}, y_{0}\right)$ and control (14) we obtain

$$
\begin{aligned}
y(k)-x(k)= & B^{k} y_{0}-A^{k} x_{0} \\
& +\sum_{i=1}^{k}\left(B^{k-i} v(i)-A^{k-i}\left(F\left(k_{1}, i\right) v(i)+\widehat{w}\right)\right) \\
= & B^{k} y_{0}-A^{k} x_{0}+\sum_{i=1}^{k}\left(B^{k-i}-A^{k-i} F\left(k_{1}, i\right)\right) v(i) \\
& +\sum_{i=1}^{k} A^{k-i} \widehat{w} .
\end{aligned}
$$

In Case 1 , since $\widehat{w}$ is a solution of (13), it follows from (17) at $k=k_{1}$ that

$$
\begin{aligned}
- & m_{1}+y\left(k_{1}\right)-x\left(k_{1}\right) \\
= & -m_{1}+B^{k_{1}} y_{0}-A^{k_{1}} x_{0} \\
& -\left(A^{k_{1}-1}+A^{k_{1}-2}+\cdots+A+E\right) \widehat{w} \\
& +\sum_{i=1}^{k_{1}}\left(B^{k_{1}-i}-A^{k_{1}-i} F\left(k_{1}, i\right)\right) v(i) \\
= & \sum_{i=1}^{k_{1}}\left(B^{k_{1}-i}-A^{k_{1}-i} F\left(k_{1}, i\right)\right) v(i) .
\end{aligned}
$$

Next, if pursuit is not completed in the game (4), (5) at the step $k_{1}$, then in view of (18) we obtain

$$
\begin{aligned}
\mid- & m_{1}+y\left(k_{1}\right)-x\left(k_{1}\right) \mid \\
& =\left|\sum_{i=1}^{k_{1}}\left(B^{k_{1}-i}-A^{k_{1}-i} F\left(k_{1}, i\right)\right) v(i)\right|>\ell .
\end{aligned}
$$

Then using the Cauchy-Schwartz inequality and condition (5) (a) of Assumption 3 yields

$$
\begin{aligned}
\ell & <\left|\sum_{i=1}^{k_{1}}\left(B^{k_{1}-i}-A^{k_{1}-i} F\left(k_{1}, i\right)\right) v(i)\right| \\
& <\left(\sum_{i=1}^{k_{1}}\left\|B^{k_{1}-i}-A^{k_{1}-i} F\left(k_{1}, i\right)\right\|^{2}\right)^{1 / 2} \cdot\left(\sum_{i=1}^{k_{1}}|v(i)|^{2}\right)^{1 / 2} \\
& \leq\left(d \cdot m^{\alpha_{1}}\right)^{1 / 2} \cdot\left(\sum_{i=1}^{k_{1}}|v(i)|^{2}\right)^{1 / 2} .
\end{aligned}
$$

Hence,

$$
\sum_{i=1}^{k_{1}}|v(i)|^{2}>\frac{\ell^{2}}{\left(d \cdot m^{\alpha_{1}}\right)}
$$

Clearly, if inequality (19) is not satisfied, then pursuit starting from the initial position $z_{0}=\left(x_{0}, y_{0}\right), y_{0}-x_{0} \notin M$, is completed on the step $k_{1}$ in the game (4), (5). Therefore, assuming that pursuit is not completed up to the step $k_{1}$ in the game (4), (5), which implies inequalities (19) and (21), we conclude that the evader must expend the resources greater than $\ell^{2} /\left(d \cdot m^{\alpha_{1}}\right)$ for the steps $1, \ldots, k_{1}$.

In Case 2 , the evader expends resources equal to $\sigma^{2} /\left(m^{1-2 \alpha_{2}}\right)$ for the steps $1, \ldots, n_{1}$.

We turn to Case 3 . Since inequality (15) is satisfied at $k=$ $n_{1}-1$ and (16) is satisfied at $k=n_{1}$, then

$$
\sum_{i=1}^{n_{1}-1}|v(i)|^{2}+\frac{1}{d_{1}^{2}}\left|v\left(n_{1}\right)\right|^{2}=\frac{\sigma^{2}}{m^{1-2 \alpha_{2}}}
$$


at some $d_{1}>1$. Define the value $u\left(n_{1}\right)$ of the pursuer's control as follows:

$$
u\left(n_{1}\right)=F\left(k_{1}, n_{1}\right)\left(\frac{1}{d_{1}} v\left(n_{1}\right)\right)+\widehat{w} .
$$

Admissibility of the pursuer's control will be shown later. Thus, in Case 3, to guarantee that pursuit is not completed up to the step $n_{1}$, the evader must expend resources greater than $\sigma^{2} / m^{1-2 \alpha_{2}}$.

The process of the game up to the step $k_{1}$ in Case 1 and up to the step $n_{1}$ in Cases 2 and 3 will be referred to as the process of first approach of the pursuer to evader.

We now calculate the amount of resources expended by the pursuer on the process of the first approach.

In Case 1, using the Minkowski inequality, we obtain from (14) that

$$
\begin{aligned}
\sum_{i=1}^{k_{1}}|u(i)|^{2} & =\sum_{i=1}^{k_{1}}\left|F\left(k_{1}, i\right) v(i)+\widehat{w}\right|^{2} \\
& \leq\left[\left(\sum_{i=1}^{k_{1}}\left|F\left(k_{1}, i\right) v(i)\right|^{2}\right)^{1 / 2}+\left(\sum_{i=1}^{k_{1}}|\widehat{w}|^{2}\right)^{1 / 2}\right]^{2} .
\end{aligned}
$$

In Case 2, in view of (14) we have

$$
\begin{aligned}
\sum_{i=1}^{n_{1}}|u(i)|^{2} & =\sum_{i=1}^{n_{1}}\left|F\left(k_{1}, i\right) v(i)+\widehat{w}\right|^{2} \\
& \leq\left[\left(\sum_{i=1}^{n_{1}}\left|F\left(k_{1}, i\right) v(i)\right|^{2}\right)^{1 / 2}+\left(\sum_{i=1}^{n_{1}}|\widehat{w}|^{2}\right)^{1 / 2}\right]^{2} .
\end{aligned}
$$

In Case 3, we use (14) and (23) to estimate the resources of the pursuer. Since $d_{1}>1$, using the Minkowski inequality, we see that

$$
\begin{aligned}
\sum_{i=1}^{n_{1}}|u(i)|^{2}= & \sum_{1}^{n_{1}-1}\left|F\left(k_{1}, i\right) v(i)+\widehat{w}\right|^{2} \\
& +\left|F\left(k_{1}, n_{1}\right)\left(\frac{1}{d_{1}} v\left(n_{1}\right)\right)+\widehat{w}\right|^{2} \\
\leq & {\left[\left(\sum_{i=1}^{n_{1}-1}\left|F\left(k_{1}, i\right) v(i)\right|^{2}\right.\right.} \\
& \left.\left.\left.+\left(\sum_{i=1}^{n_{1}}|\widehat{w}|^{2}\right)^{1 / 2}\right]^{2}\right]^{2}\left|F\left(k_{1}, n_{1}\right) v\left(n_{1}\right)\right|^{2}\right)^{1 / 2} \\
\leq & \left(\left(\sum_{i=1}^{n_{1}}\left|F\left(k_{1}, i\right) v(i)\right|^{2}\right)^{1 / 2}+\left(\sum_{i=1}^{n_{1}}|\widehat{w}|^{2}\right)^{1 / 2}\right)^{2} .
\end{aligned}
$$

Similarly, according to condition (5) (b) of Assumption 3 and the fact that $\widehat{w} \in\left(\rho_{1} / \sqrt{m k_{1}}\right) S, n_{1}<k_{1}$, the right-hand sides of inequalities (24)-(26) can be estimated from the above by $\rho^{2} / m$. For example, the right-hand side of (24) is estimated as follows:

$$
\begin{gathered}
{\left[\left(\sum_{i=1}^{k_{1}}\left\|F\left(k_{1}, i\right)\right\|^{2} \cdot|v(i)|^{2}\right)^{1 / 2}+\left(\sum_{i=1}^{k_{1}}|\widehat{w}|^{2}\right)^{1 / 2}\right]^{2}} \\
\leq\left[\frac{\rho_{2}}{\sigma m^{\alpha_{2}}}\left(\sum_{i=1}^{k_{1}}|v(i)|^{2}\right)^{1 / 2}+\frac{\rho_{1}}{\sqrt{m}}\right]^{2} \\
\leq\left(\frac{\rho_{2}}{\sigma \cdot m^{\alpha_{2}}} \cdot \frac{\sigma}{\sqrt{m^{1-2 \alpha_{2}}}}+\frac{\rho_{1}}{\sqrt{m}}\right)^{1 / 2}=\frac{\rho^{2}}{m} .
\end{gathered}
$$

Therefore,

$$
\begin{gathered}
\sum_{i=1}^{k_{1}}|u(i)|^{2} \leq \frac{\rho^{2}}{m} \quad \text { in Case 1, } \\
\sum_{i=1}^{n_{1}}|u(i)|^{2} \leq \frac{\rho^{2}}{m} \quad \text { in Cases } 2 \text { and } 3 .
\end{gathered}
$$

We conclude from these estimations that if pursuit is not completed in the game (4), (5) during the process of first approach, the amount of resources expended by the evader is greater than or equal to $\min \left\{\sigma^{2} / m^{1-2 \alpha_{2}}, \ell^{2} /\left(d \cdot m^{\alpha_{1}}\right)\right\}$, and that expended by the pursuer does not exceed $\rho^{2} / m$.

Further, we continue as follows. In Case 1, the points $x_{01}=$ $x\left(k_{1}\right), y_{01}=y\left(k_{1}\right)$ will be taken as the initial position at $k_{1}$, and in Cases 2 and 3, the points $x_{01}=x\left(n_{1}\right), y_{01}=y\left(n_{1}\right)$ will be taken as the initial position at $n_{1}$. We now consider (4), (5) with the initial position $\left(x_{01}, y_{01}\right)$ for the steps $k=1,2, \ldots$

Given the initial position $\left(x_{01}, y_{01}\right), y_{01}-x_{01} \notin M$, we find the least integer $k_{2}=k_{2}\left(x_{01}, y_{01}\right)>0$ such that

$$
\begin{aligned}
-m_{2}+B^{k_{2}} y_{01}-B^{k_{2}} x_{01} & \in \frac{\rho_{1}}{\sqrt{m k_{2}}} \\
& \times\left(A^{k_{2}-1}+A^{k_{2}-2}+\cdots+A+E\right) S,
\end{aligned}
$$

at some $m_{2} \in M$. Existence of such $k_{2}$ is guaranteed by condition (1) of Assumption 3.

Let $v=v(i), 1 \leq i \leq k_{2}$, be an arbitrary control of the evader. For the steps $i \in\left\{1, \ldots, k_{2}\right\}$, we define the control of the pursuer $u=u(i)$ by (14), with $k_{1}$ replaced by $k_{2}$ while the inequality (15) holds.

We apply the above argument again. If pursuit is not completed in the game (4), (5) during the process of second approach, then the amount of resources expended by the evader is not less than $\min \left\{\sigma^{2} / m^{1-2 \alpha_{2}}, \ell^{2} /\left(d \cdot m^{\alpha_{1}}\right)\right\}$, and that expended by the pursuer is less than $\rho^{2} / m$.

Now, $m$-time repeated application of this reasoning enables us to conclude that at most at the $m$ th process of approach pursuit is completed in the game (4), (5). 
Indeed, assuming the contrary we have

$$
\begin{aligned}
\sigma^{2} & \geq \sum_{j=1}^{m}\left(\sum_{i=1}^{k_{j}}|v(i)|^{2}\right) \geq \sum_{j=1}^{m} \min \left\{\frac{\ell^{2}}{d \cdot m^{\alpha_{1}}}, \frac{\sigma^{2}}{m^{1-2 \alpha_{2}}}\right\} \\
& =m \cdot \min \left\{\frac{\ell^{2}}{d \cdot m^{\alpha_{1}}}, \frac{\sigma^{2}}{m^{1-2 \alpha_{2}}}\right\},
\end{aligned}
$$

which contradicts condition (3) of Assumption 3.

However for $u=u(\cdot)$ we have $\sum_{j=1}^{m} \sum_{i=1}^{k_{j}}|u(i)|^{2} \leq \sum_{j=1}^{m}$ $\left(\rho^{2} / m\right)=\rho^{2}$. The proof is complete.

Remark 5. Let condition (2) of Assumption 3 hold for $m=1$ and $d<\ell^{2} / \sigma^{2}$. Then conditions (1) and (3) of Assumption 3 can be weakened. More precisely, we require the inclusion $y_{0}-x_{0} \in M$ and drop condition (3). Then Theorem 4 guarantees that pursuit can be completed only from the initial points $x_{0}, y_{0}$.

Example 6. Consider a discrete game described by equations

$$
\begin{aligned}
& x_{k}=\lambda x_{k-1}+u_{k}, \\
& y_{k}=\lambda y_{k-1}+v_{k},
\end{aligned}
$$

where $x_{k}, y_{k}, u_{k}, v_{k} \in R^{n}, n \geq 1$. Let $u=\left(u_{1}, u_{2}, \ldots, u_{k}, \ldots\right)$, $v=\left(v_{1}, v_{2}, \ldots, v_{k}, \ldots\right)$, and

$$
\|u(\cdot)\|_{l_{2}} \leq \rho, \quad\|v(\cdot)\|_{l_{2}} \leq \sigma .
$$

The terminal set is $M=\left\{x, y \mid x, y \in R^{n}, y-x \in l S, l>\right.$ $0\}$. For this example, $B=A=\lambda E$. Applying Theorem 4 to the game (31) and (32), we obtain the following statement.

Assertion 1. If $\rho>\sigma$ and $|\lambda| \leq 1$, then pursuit can be completed in the game (31) and (32) from any initial positions in finite number of steps.

Proof of assertion is straightforward if we take $F(k, i)=E$, $m=1, \rho=\rho_{1}+\rho_{2}, \rho_{1}>0, \rho_{2}>\sigma$, and $\alpha_{1}=\alpha_{2} \neq 0$, and $d>$ 0 is sufficiently small number. For Assertion 1 we used Remark 5 .

\section{Conclusion}

We have obtained sufficient conditions of completion of pursuit for a linear pursuit discrete game with total constraints where motions of the players are described by different-type linear equations. The control parameter of the pursuer $u(k)$ is constructed based on $v(k)$.

It should be noted that conclusion of Theorem 4 is still true with appropriate changes if controls $u=u(\cdot)$ and $v=$ $v(\cdot)$ are subjected to constraints $\|u(\cdot)\|_{l_{p}} \leq \rho$ and $\|v(\cdot)\|_{l_{p}} \leq \sigma$, $p>1$. Further studies can be done to weaken conditions of Assumption 3.

\section{Conflict of Interests}

The authors declare that there is no conflict of interests regarding the publication of this paper.

\section{Acknowledgment}

The present research was partially supported by the National Fundamental Research Grant Scheme (FRGS) of Malaysia, 01-01-13-1228FR.

\section{References}

[1] A. Ya. Azimov and G. Kh. Guseinov, "On some classes of differential games with integral constraints," Izvestiya Akademii Nauk SSSR. Tekhnicheskaya Kibernetika, no. 3, pp. 9-16, 1972.

[2] A. A. Chikrii and A. A. Belousov, "On linear differential games with integral constraints. Memoirs of Institute of Mathematics and Mechanics," Ural Branch of the Russian Academy of Sciences, Ekaterinburg, vol. 15, no. 4, pp. 290-301, 2009.

[3] G. I. Ibragimov, "On the problem of group pursuit with integral constraints on the controls of the players," Rossiuskaya Akademiya Nauk. Matematicheskie Zametki, vol. 70, no. 2, pp. 201-212, 2001.

[4] G. I. Ibragimov and N. Y. Satimov, "A multiplayer pursuit differential game on a closed convex set with integral constraints," Abstract and Applied Analysis, vol. 2012, Article ID 460171, 12 pages, 2012.

[5] R. Isaacs, Differential Games. A Mathematical Theory with Applications to Warfare and Pursuit, Control and Optimization, New York, NY, USA, 1967.

[6] N. N. Krasovskii, The Theory of Motion Control, Nauka, Moscow, Russia, 1970.

[7] N. N. Krasovskiǐ and A. I. Subbotin, Game-Theoretical Control Problems, Springer, New York, NY, USA, 1988.

[8] A. V. Mezentsev, Differential Games with Integral Constraints, Michigan State University, East Lansing, Mich, USA, 1988.

[9] M. S. Nikolskii, "A direct method in linear differential games with integral constraints," in Controlled Systems, vol. 2, pp. 49-59, Institut Kataliza Sibirskogo Otdeleniya Akademii Nauk SSSR, Novosibirsk, Russia, 1969.

[10] L. S. Pontryagin, Selected Works, Nauka, Moscow, Russia, 1988.

[11] A. T. Rahmanov, "Research of pursuit differential games with geometric and integral constraints," Reports of Academy of Sciences of Uzbekistan, no. 10, pp. 3-5, 1986.

[12] A. T. Rakhmanov, "A pursuit method in linear differential games with integral constraints on the controls," Differential Equations, vol. 25, no. 5, pp. 785-790, 1989.

[13] B. T. Samatov, "Problems of group pursuit with integral constraints on controls of the players," Cybernetics and Systems Analysis, vol. 49, no. 5, pp. 756-767, 2013.

[14] N. Y. Satimov, B. B. Rikhsiev, and A. A. Hamdamov, "On A Pursuit Problem for n-person linear differential and discrete games with integral constraints," Mathematics of the USSR-Sbornik, vol. 46, no. 4, pp. 456-469, 1983.

[15] N. Y. Satimov, A. Z. Fazylov, and A. A. Khamdamov, "Problems of pursuit and evasion in differential and discrete $n$-person games with integral constraints," Differential Equations, vol. 20, no. 8, pp. 1388-1396, 1984.

[16] N. Y. Satimov and G. I. Ibragimov, "On a pursuit problem for discrete games with several participants," Izvestiya Vysshikh Uchebnykh Zavedeniu. Matematika, no. 12511, pp. 46-57, 2004.

[17] N. Y. Satimov, Methods to Solve Pursuit Problems in DifferentialGame Theory, NUU Press, Tashkent, Uzbekistan, 2003.

[18] A. I. Subbotin and A. G. Chentsov, Optimization of the Guarantee in Control Problems, Nauka, Moscow, Russia, 1981. 
[19] A. A. Azamov and A. Sh. Kuchkarov, "Controllability and pursuit problems in linear discrete systems," Journal of Computer and Systems Sciences International, no. 3, pp. 21-26, 2010.

[20] G. I. Ibragimov, "Problems of linear discrete games of pursuit," Mathematical Notes, vol. 77, no. 5, pp. 653-662, 2005.

[21] G. I. Ibragimov and A. Sh. Kuchkarov, "Discrete pursuit game with total constraints," in Proceedings of the International Symposium on New Development of Geometric Function Theory and its Applications, pp. 370-374, Selangor, Malaysia, November 2008.

[22] A. Kuchkarov, G. Ibragimov, and A. Sotvoldiev, "Linear discrete pursuit game problem with total constraints," Abstract and Applied Analysis, vol. 2013, Article ID 840925, 5 pages, 2013. 


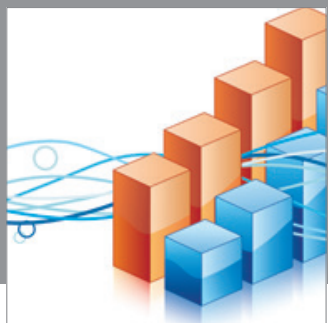

Advances in

Operations Research

mansans

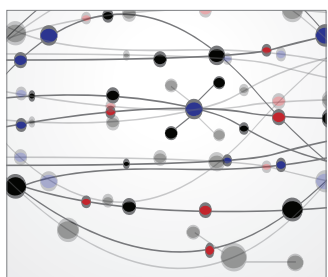

The Scientific World Journal
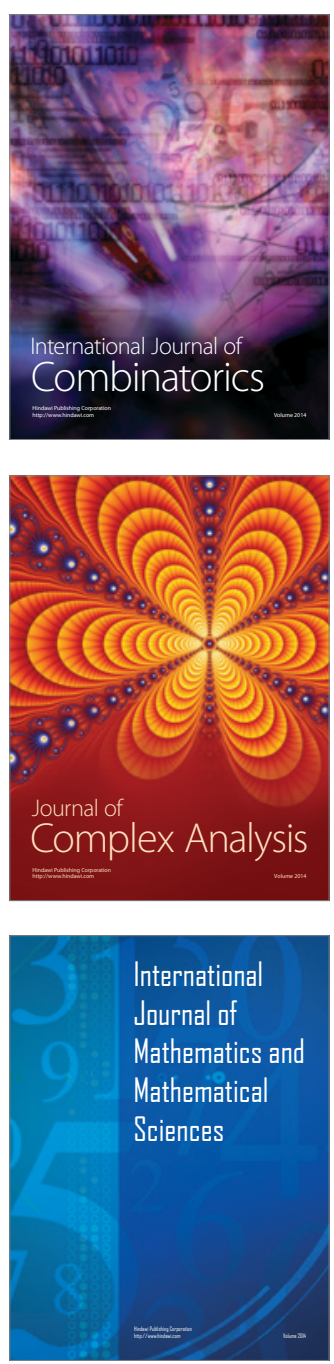
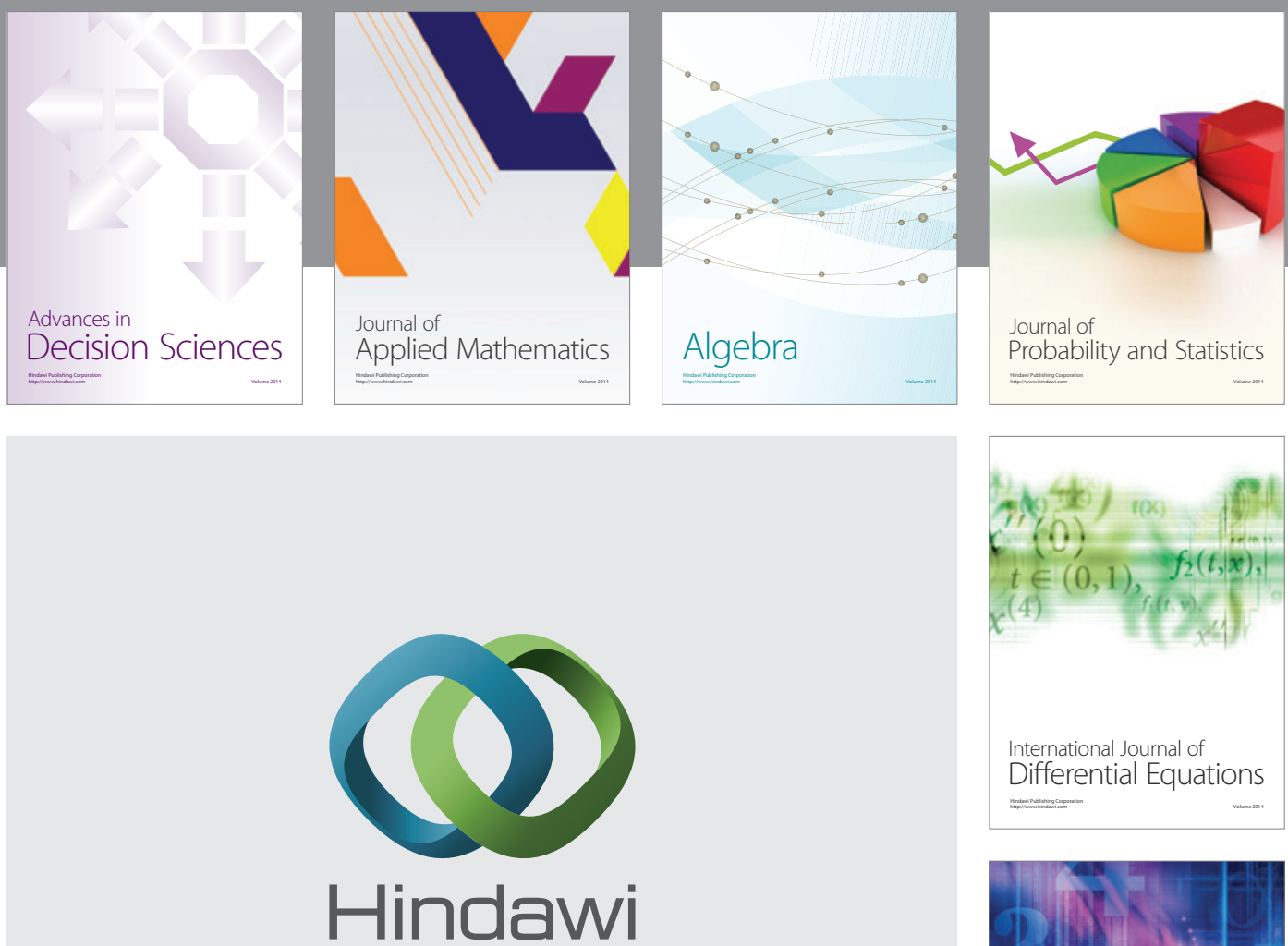

Submit your manuscripts at http://www.hindawi.com
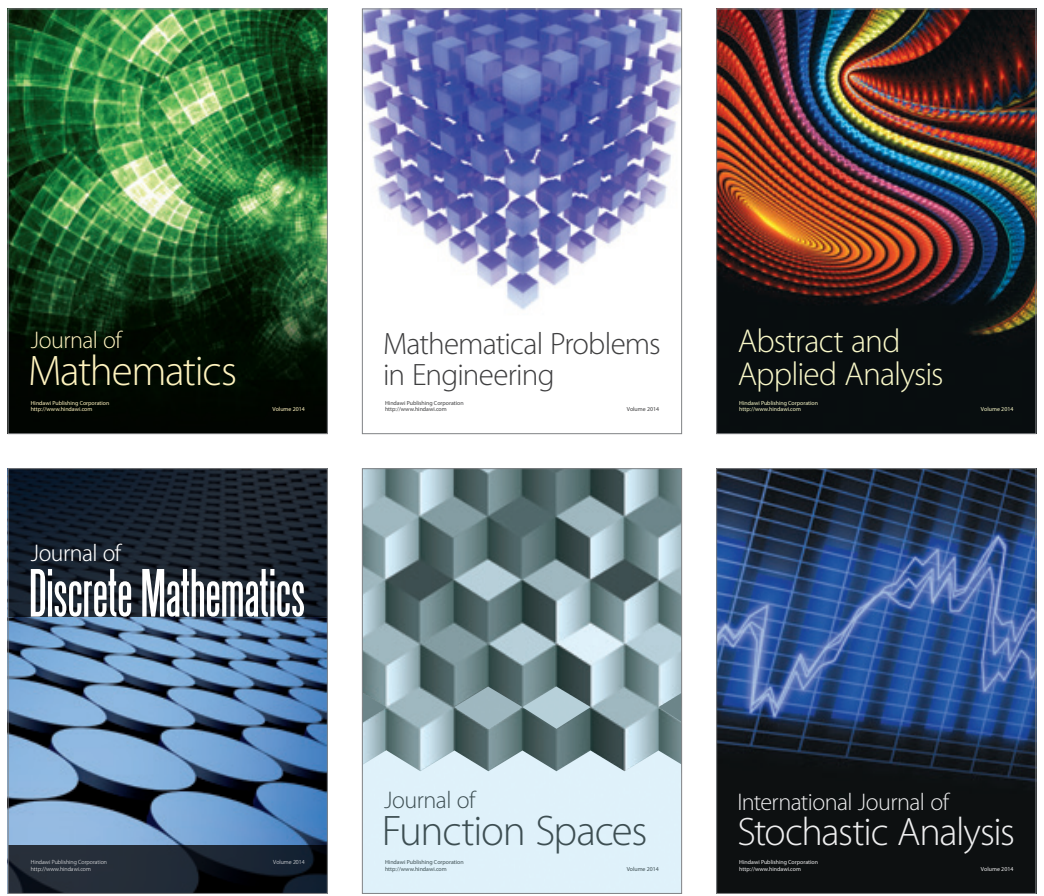

Journal of

Function Spaces

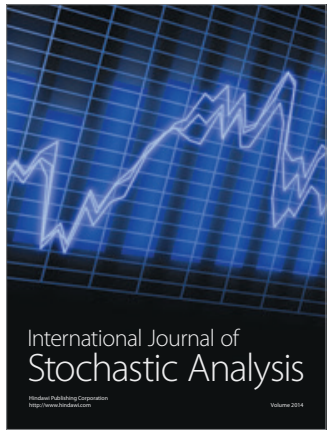

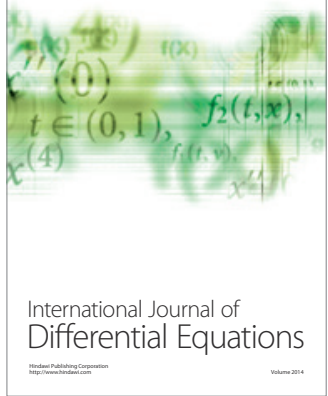
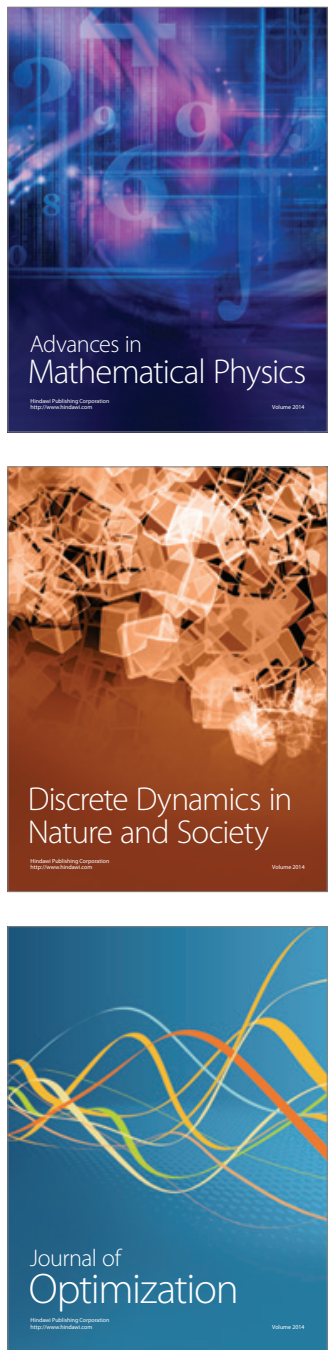